\title{
Dog keeping at home before and during pregnancy decreased the risk of food allergy in 1-year-old children
}

\author{
Katarzyna Smejda ${ }^{1}$, Kinga Polanska², Wlodzimierz Stelmach³, Pawel Majak ${ }^{4}$ Iwona Stelmach ${ }^{1}$ \\ ${ }^{1}$ Department of Paediatrics and Allergy, Medical University of Lodz, Copernicus Memorial Hospital, Lodz, Poland \\ 2Department of Environmental Epidemiology, Nofer Institute of Occupational Medicine, Lodz, Poland \\ ${ }^{3}$ Department of Social and Preventive Medicine, Medical University of Lodz, Lodz, Poland \\ ${ }^{4}$ Department of Internal Medicine, Asthma and Allergy, Medical University of Lodz, Lodz, Poland
}

Adv Dermatol Allergol 2020; XXXVII (2): 255-261

DOI: https://doi.org/10.5114/ada.2018.80584

\begin{abstract}
Introduction: The relationship between allergen exposure to animals in pregnancy and the development of allergic symptoms is not clear.

Aim: To evaluate the association between prenatal and postnatal exposure to pet ownership and development of atopic dermatitis, food allergy and wheezing in children at the age of 1 and 2.

Material and methods: The mother-child pairs included in this study were part of the Polish Mother and Child Cohort. Mothers in each trimester of pregnancy and 1 year after childbirth have completed a questionnaire on animal exposure. Children's health status was assessed at around one year and two years of age.

Results: Keeping a dog at home before and during pregnancy (every trimester) decreased the risk of food allergy in the first year of life. On the other hand, keeping any animal other than a dog (cat, hamster, guinea pig, rabbit) before pregnancy and during each trimester separately increased the risk of food allergy in the first year of life of children. Keeping a guinea pig in the first trimester of pregnancy increased the risk of wheezing in the first year of life. The analysis did not show any significant associations between keeping animals at home before and during pregnancy and the occurrence of atopic dermatitis in the second year of life.

Conclusions: Keeping a dog at home before and during pregnancy decreased the risk of food allergy in 1-year-old children. This effect was eliminated in case of having a cat, hamster, guinea pig, or rabbit.
\end{abstract}

Key words: children, pet, allergic diseases.

\section{Introduction}

Exposure to animal allergens is a major risk factor for the development of sensitization and allergic diseases [1-4]. Cats, dogs, guinea pigs, hamsters, and rabbits are very popular pets in industrialized countries, where the percentage of pet ownership continues to increase [5]. Cats and dogs are the most common pets living indoor environments and the frequency of their ownership is highly variable in Europe ranging from $7.2 \%$ to $35 \%$ for the cats and from 5.4 to $35 \%$ for the dogs [6]. In Poland these numbers are even higher; a survey from 2014 shows that $83 \%$ of Poles have a dog in their home and $44 \%$ own a cat, $4 \%$ - birds, $3 \%$ - rodents (mice, rats, hamsters) and $1 \%$ - reptiles [7].
The percentage of pet allergens carried on small particles (about 0.5-2 $\mu \mathrm{m}$ ) become easily airborne under normal domestic ventilation and are able to trigger respiratory symptoms in sensitized patients. These allergens in the lower airways can cause prolonged bronchial inflammation [8], leading to the potential for acute airway tightening/obstruction [9, 10]. Cat and dog allergens should be considered as ubiquitous because they are found not only at homes, but also in other indoor private or public places where cats/dogs have been never kept [11]. Accumulation of pet allergens in indoor environments without animals correlate with the number of visitors owning a pet or with those who are in regular contact with these animals [11]. Several authors have shown that clothing and other items, such as human hair, con-

Address for correspondence: Prof. Iwona Stelmach MD, PhD, Department of Paediatrics and Allergy, Medical University of Lodz, Copernicus Memorial Hospital, Korczak Paediatric Centre, 71 Piłsudskiego St, 90-329 Lodz, Poland, phone: +48 42 207 4726 , fax: +48 4267763 58, e-mail: alergol@kopernik.lodz.pl Received: 15.10.2018, accepted: 31.10.2018. 
stitute a means for transferring pet allergens in pet-free indoor environments [12-14]. Therefore, the higher the pet ownership in a given community, the higher the presence of pet allergens in apparently pet-free spaces [15].

According to some authors, children with asthma tend to become sensitized to the allergens prominent in their living environments. Sheikh et al. suggest a positive association between life style choices/environmental exposures and family history of asthma [16].

\section{Aim}

The aim of the present study was to evaluate the association between prenatal and postnatal exposure to pet ownership and development of atopic dermatitis, food allergy and wheezing in children aged 1 and 2 .

\section{Material and methods}

\section{Study design and population}

The mother-child pairs included in this study were part of the Polish Mother and Child Cohort (REPRO_PL) - a multicentre prospective cohort established in 2007 with the aim to evaluate environmental factors contributing to the pregnancy outcomes and children's health [17-19]. All data were prospectively collected. Women were recruited if they fulfilled the following inclusion criteria: single pregnancy up to 12 weeks of gestation, no assisted conception, no pregnancy complications, and no chronic diseases as specified in the study protocol [17, 19]. The whole cohort comprises 1700 mother-child pairs followed-up from the pregnancy period [20]. The present assessment is restricted to subpopulation from the Lodz district (central Poland).

The participants were interviewed once in each trimester of pregnancy to collect and update socio-demographic data, medical and reproductive history, information about environmental, occupational and lifestyle factors. Children were assessed for their exposure and health status at 1 year of age [18, 21-24].

The study was approved by the Ethical Committee of the Nofer Institute of Occupational Medicine, Lodz, Poland; written consent was obtained from all the subjects before the study.

\section{Assessment of maternal exposure to animals during pregnancy}

Mothers in each trimester of pregnancy have completed a questionnaire on animal exposure (having a pet at home), including exposure to pets for 3 months before becoming pregnant. In addition, a questionnaire with mothers was conducted one year after childbirth. The questions concerned: a) having pets in the house where the baby was, and b) the species of the pet possessed (cat, dog, rabbit, hamster, guinea pig).

\section{Child health assessment}

Children's health status was assessed at around 1 year and 2 years of age. For the appropriate recognition of the children's health status, an interview was performed with mothers and supplemented (verified) with information from the medical chart of each child [18, 21-25]. In addition, the current child health status assessment was performed by a paediatrician/allergist. Finally, based on availability of the data on exposure and physical examination at the age of 24 months, the analysis was performed for 529 children.

The first part of the questionnaire covers certain sociodemographic information (i.e., family size, financial status of the family, and parental educational level). The occurrence of allergy among family members was noted. The second part of the questionnaire (developed by an allergist, based on recommendations from the International Study of Asthma and Allergies in Childhood) investigated the child's health and condition. The allergy outcomes were analysed for the following symptoms: wheezing, cough, bronchitis, eczema, food allergy; the childhood outcomes were confirmed by an allergist/ physician. The duration of each infection and disease, medications taken, and hospitalizations, if any, were identified.

\section{Statistical analysis}

The associations between presence of: i) atopic dermatitis, ii) food allergy, iii) at least one episode of wheezing (defined as dependent variables) and animal exposure (defined as independent variable) were assessed by logistic regression. The effect of animals exposure was corrected for the effects of other independent risk factors of dependent variable, defined previously in this cohort. First, logistic regression was used to assess the relationship between dependent variables and each of the independent variables (presented in Tables 1 and 2). A stepwise forward procedure was then used to select variables. Predictors with $p$ levels of at least 0.1 estimated in univariate models were included into multivariate regression analyses. All of the statistical analyses were performed using SPSS 11.5. The null hypothesis was rejected if $p<0.05$.

\section{Results}

Five hundred and thirty nine participants were included in the current analysis. Baseline characteristics of these population are given in Table 3. The lifetime prevalence of the selected outcomes is given. Almost $40 \%$ of children had a positive parental history of atopy.

The results of the univariate logistic regression are presented in Tables 1 and 2. Associations between keeping a pet at home before pregnancy and every trimester and doctor-diagnosed food allergy was found. Keeping 
Table 1. Associations between presence of atopic dermatitis, food allergy, wheezing (at least one episode) during the first year of life and animal exposure at home during pregnancy in univariate model of logistic regression analysis

\begin{tabular}{|c|c|c|c|c|c|c|c|c|c|}
\hline \multirow[t]{2}{*}{ Animal exposure } & \multicolumn{3}{|c|}{ Atopic dermatitis } & \multicolumn{3}{|c|}{ Food allergy } & \multicolumn{3}{|c|}{ Wheezing } \\
\hline & OR & \multicolumn{2}{|c|}{$95 \% \mathrm{Cl}$ for OR } & OR & \multicolumn{2}{|c|}{$95 \% \mathrm{Cl}$ for $\mathrm{OR}$} & OR & \multicolumn{2}{|c|}{$95 \% \mathrm{Cl}$ for $\mathrm{OR}$} \\
\hline Cat & 1.06 & 0.59 & 1.92 & 0.79 & 0.48 & 1.28 & 0.96 & 0.49 & 1.89 \\
\hline Dog & 0.63 & 0.36 & 1.13 & 0.48 & 0.30 & 0.76 & 1.70 & 0.83 & 3.49 \\
\hline Hamster & 0.95 & 0.11 & 8.14 & 0.53 & 0.06 & 4.72 & 3.94 & 0.68 & 22.72 \\
\hline Guinea pig & - & - & - & 1.23 & 0.23 & 6.42 & 4.56 & 0.71 & 29.12 \\
\hline Any animal & 1.30 & 0.69 & 2.46 & 1.93 & 1.18 & 3.16 & 0.71 & 0.22 & 2.26 \\
\hline \multicolumn{10}{|l|}{ First trimester: } \\
\hline Cat & 0.84 & 0.45 & 1.58 & 0.88 & 0.54 & 1.45 & 0.97 & 0.48 & 1.95 \\
\hline Dog & 0.74 & 0.42 & 1.31 & 0.48 & 0.31 & 0.76 & 1.48 & 0.76 & 2.91 \\
\hline Hamster & 1.80 & 0.36 & 8.96 & 1.22 & 0.23 & 6.56 & 2.99 & 0.56 & 16.06 \\
\hline Guinea pig & - & - & - & 1.82 & 0.32 & 10.34 & 10.32 & 1.65 & 64.47 \\
\hline Any animal & 1.24 & 0.68 & 2.27 & 1.97 & 1.24 & 3.13 & 0.83 & 0.30 & 2.29 \\
\hline \multicolumn{10}{|l|}{ Second trimester: } \\
\hline Cat & 1.00 & 0.52 & 1.90 & 1.10 & 0.66 & 1.83 & 0.67 & 0.30 & 1.47 \\
\hline Dog & 0.60 & 0.33 & 1.07 & 0.59 & 0.37 & 0.93 & 1.41 & 0.72 & 2.75 \\
\hline Hamster & - & - & - & 0.62 & 0.07 & 5.48 & 3.54 & 0.62 & 20.24 \\
\hline Guinea pig & 0.85 & 0.10 & 7.13 & 2.33 & 0.60 & 8.98 & 3.20 & 0.59 & 17.34 \\
\hline Any animal & 1.48 & 0.82 & 2.65 & 1.64 & 1.04 & 2.60 & 0.78 & 0.38 & 1.59 \\
\hline \multicolumn{10}{|l|}{ Third trimester: } \\
\hline Cat & 0.98 & 0.52 & 1.85 & 1.22 & 0.73 & 2.03 & 0.66 & 0.30 & 1.49 \\
\hline Dog & 0.56 & 0.32 & 1.00 & 0.53 & 0.33 & 0.84 & 1.79 & 0.89 & 3.60 \\
\hline Hamster & - & - & - & 1.61 & 0.14 & 18.33 & 3.68 & 0.32 & 42.45 \\
\hline Guinea pig & - & - & - & 1.25 & 0.23 & 6.65 & 3.52 & 0.61 & 20.49 \\
\hline Any animal & 1.62 & 0.92 & 2.86 & 1.74 & 1.09 & 2.78 & 0.66 & 0.32 & 1.38 \\
\hline
\end{tabular}

- Not enough observation to calculate OR (95\% CI).

a dog at home before and during pregnancy (every trimester) decreased the risk of food allergy in the first year of life (OR; 95\% CI) (Table 1). On the other hand, keeping any animal other than a dog (cat, hamster, guinea pig, rabbit) before pregnancy and during each trimester separately increased the risk of food allergy in the first year of life (OR; 95\% Cl) (Table 1).

We found an association between keeping a guinea pig in the first trimester of pregnancy and increasing the risk of wheezing in the first year of life (OR; 95\% Cl) (Table 1).

The analysis did not show any significant associations between keeping animals at home before and during pregnancy and the occurrence of atopic dermatitis in the second year of life (OR; 95\% Cl) (Table 2).

All the above associations were still significant after adjustment in the multivariate model. Independent statistical predictors of study outcomes determined previously in this cohort were used. The adjustment variables for atopic dermatitis were: atopy in the family, father's education, frequency of cleaning, and breastfeeding. Associations with food allergy are corrected for breastfeeding. The number of infections and paternal/maternal smoking were used as adjustment variables for wheezing.

\section{Discussion}

Exposure to animal allergens constitutes a relevant risk factor for the development of allergic sensitization. We showed that keeping a dog at home before and during pregnancy decreased the risk of food allergy in the first year of life. On the other hand, keeping any animal other than a dog (cat, hamster, guinea pig, rabbit) before and during pregnancy separately increased the risk of food allergy in the first year of life; having a guinea pig in the first trimester of pregnancy increased the risk of wheezing in the first year of life. Our analysis did not show any significant associations between keeping ani- 
Table 2. Associations between presence of atopic dermatitis, food allergy, wheezing (at least one episode) during the second year of life and animal exposure at home during pregnancy in univariate model of logistic regression analysis

\begin{tabular}{|c|c|c|c|c|c|c|c|c|c|}
\hline \multirow[t]{2}{*}{ Animal exposure } & \multicolumn{3}{|c|}{ Atopic dermatitis } & \multicolumn{3}{|c|}{ Food allergy } & \multicolumn{3}{|c|}{ Wheezing } \\
\hline & OR & \multicolumn{2}{|c|}{$95 \% \mathrm{Cl}$ for $\mathrm{OR}$} & OR & \multicolumn{2}{|c|}{$95 \% \mathrm{Cl}$ for $\mathrm{OR}$} & OR & \multicolumn{2}{|c|}{$95 \% \mathrm{Cl}$ for OR } \\
\hline Cat & 0.75 & 0.39 & 1.44 & 0.55 & 0.28 & 1.08 & 0.90 & 0.45 & 1.82 \\
\hline Dog & 1.05 & 0.57 & 1.96 & 0.92 & 0.49 & 1.71 & 1.20 & 0.60 & 2.41 \\
\hline Hamster & 2.04 & 0.18 & 23.04 & 1.99 & 0.17 & 22.96 & 2.79 & 0.25 & 31.66 \\
\hline Guinea pig & 1.15 & 0.12 & 10.82 & - & - & - & 1.43 & 0.15 & 13.45 \\
\hline Any animal & 1.11 & 0.57 & 2.15 & 1.51 & 0.79 & 2.90 & 1.00 & 0.48 & 2.09 \\
\hline \multicolumn{10}{|l|}{ First trimester: } \\
\hline Cat & 0.99 & 0.52 & 1.91 & 0.88 & 0.45 & 1.73 & 0.96 & 0.47 & 1.96 \\
\hline Dog & 0.92 & 0.50 & 1.70 & 0.84 & 0.45 & 1.58 & 1.30 & 0.65 & 2.59 \\
\hline Hamster & 4.17 & 0.57 & 30.37 & 4.35 & 0.60 & 31.78 & 1.96 & 0.20 & 19.44 \\
\hline Guinea pig & - & - & - & - & - & - & 2.56 & 0.22 & 29.87 \\
\hline Any animal & 1.04 & 0.55 & 1.93 & 1.27 & 0.68 & 2.39 & 0.81 & 0.39 & 1.65 \\
\hline \multicolumn{10}{|l|}{ Second trimester: } \\
\hline Cat & 0.85 & 0.42 & 1.73 & 0.83 & 0.40 & 1.72 & 0.79 & 0.36 & 1.73 \\
\hline Dog & 0.91 & 0.50 & 1.66 & 0.88 & 0.48 & 1.64 & 1.14 & 0.59 & 2.22 \\
\hline Hamster & - & - & - & 2.03 & 0.18 & 23.10 & 2.64 & 0.23 & 30.19 \\
\hline Guinea pig & 4.04 & 0.55 & 29.45 & 1.38 & 0.14 & 13.66 & - & - & - \\
\hline Any animal & 0.83 & 0.45 & 1.55 & 1.00 & 0.53 & 1.86 & 1.15 & 0.60 & 2.24 \\
\hline \multicolumn{10}{|l|}{ Third trimester: } \\
\hline Cat & 0.91 & 0.46 & 1.80 & 0.98 & 0.48 & 2.01 & 1.03 & 0.47 & 2.25 \\
\hline Dog & 0.80 & 0.43 & 1.47 & 0.83 & 0.44 & 1.58 & 0.88 & 0.44 & 1.77 \\
\hline Hamster & - & - & - & 4.30 & 0.26 & 70.73 & 5.89 & 0.36 & 97.36 \\
\hline Guinea pig & - & - & - & - & - & - & - & - & - \\
\hline Any animal & 1.05 & 0.57 & 1.95 & 1.01 & 0.53 & 1.93 & 1.66 & 0.84 & 3.30 \\
\hline
\end{tabular}

- Not enough observation to calculate OR $(95 \% \mathrm{Cl})$.

mals at home before and during pregnancy and the occurrence of atopic dermatitis.

Although food allergy is the most common in infants and young children, risk factors remain largely unknown. Koplin et al. demonstrated that children with a dog at home were less likely to develop egg allergy by one year of age [26]. In our present study, we observed that having a dog before and during pregnancy reduced the risk of food allergy in the first year of life. Numerous studies suggested that dog ownership is associated with a dis tinct house dust microbial exposure [27-34]. Pets can affect the home microbiome [35]. Household dust samples collected from homes where outward-living animals were richer in bacteria and more diverse, contained more taxa in significantly higher abundance, representing the phyla Acidobacteria, Actinobacteria, Bacteroidetes, Firmicutes, Proteobacteria, Spirochaetes and Verrucomicrobia [29]. Tun et al. observed that exposure to pets increased the abundance of two bacteria, Ruminococcus and Oscillospira, which have been negatively associated with childhood atopy [36]. In particular, research suggests that pregnant mothers who live in houses with dogs are less likely to give birth to children who go on to develop allergies [32-34] or atopic dermatitis [29]. After exposure to the dust associated with the dog, home dust is added to the Lactobacillus johnsonii strains, which are associated with significant reductions in the total number and proportion of activated CD11c(+)/CD11b(+) and CD11C(+)/ CD8(+) cells [30].

Nagao et al. showed that sensitization to a dog was common at an early age despite dog ownership. Sensitization to secretoglobins and lipocalins and less to serum albumins explained the pet sensitization. Asthmatic children had a higher proportion of sensitization to dog, cat and horse allergens at entry compared with non-asthmatic children. Component resolved diagnosis 
for dog and cat allergens showed that IgE antibodies to Can $f 1$ and Fel $d 1$ was common even at a very young age [37]. Pyrhönen et al. stated that early exposure to dog and cat at home is associated with a higher incidence of respective pet allergy (slgE and skin prick tests) during the first four years of life [38]. Pohlabeln et al. showed that in families without a history of atopic disease, the prevalence of asthma and eczema among 2-year-old children was significantly lower in those families that owned a dog at the time the children were born. In contrast, in families with a history of atopic disease, early dog exposure was associated with a higher prevalence of asthma and eczema in 2-year-old children [39].

On the other hand, we have shown that having any animal (cat, hamster, guinea pig, rabbit, etc.) before and during pregnancy separately increases the risk of food allergy in the first year of life. In the case of "any animal", it is most likely the effect of summing up various factors related to the presence of animals at home. However, all individual exposures are associated with ORs below or close to 1 and then these significant associations with "any animal" and food allergy seem uncertain. Disturbance of the balance between the beneficial effect of the microbe and the adverse effect of enzymes such as proteases may increase the risk of allergy. The cat exposure in the first year of life was significantly and independently associated with current wheezing and current asthma and current rhinoconjunctivitis at the age of 7 [40]. However, Lodge et al. concluded that exposure to cats or dogs at birth showed a moderate reduction in risk of wheeze and hay fever after 7 years of age [41]. Brunekreef et al. proved among children (6-7 years of age) that cat exposure in the first year of life was associated with current symptoms of asthma, wheeze, rhinoconjunctivitis, and eczema, especially in less affluent countries [42]. Collin et al. determined that pet ownership during pregnancy and childhood in the birth cohort was consistently associated with a reduced risk of aeroallergen sensitization and atopic asthma at the age of 7 , but tended to be associated (particularly for rabbits and rodents) with an increased risk of non-atopic asthma [31].

Nordlund et al. found that a specific IgE response to more than three animal-derived components - lipocalin (nMus m 1, rEqu c 1, Fel d 4, rCan f 1, 2), kallikrein ( $r$ Can $f 5)$ and secretoglobin ( $r$ Fel d 1) - was more common among severe asthmatics compared to children with controlled asthma [43]. In another study by Eldeirawi et al., exposure in utero to cats/dogs and birds jointly was associated with increased odds of asthma, ever wheezing and current exercise-induced wheezing compared to children not exposed to these pets in utero [44].

Freitas et al. observed that the high concentration of endotoxin from Gram-negative bacteria, found in the rooms where guinea pigs were staying, was associated with increased wheezing in workers [45]. Collin et al. also confirmed that rabbit and rodent ownership in this birth
Table 3. Description of the study cohort $(n=539)$

\begin{tabular}{|c|c|}
\hline Variable & $\begin{array}{c}N(\%) \text { or } \\
\text { mean } \pm \text { SD }\end{array}$ \\
\hline \multicolumn{2}{|l|}{ Clinical profile: } \\
\hline Atopic dermatitis & $57(11)$ \\
\hline Food allergy & $56(10)$ \\
\hline Wheezing & $46(9)$ \\
\hline \multicolumn{2}{|l|}{ Family status: } \\
\hline Mother's age [year] & $28.9 \pm 4.4$ \\
\hline \multicolumn{2}{|l|}{ Maternal education: } \\
\hline$<9$ years of education & $21(4)$ \\
\hline 10-12 years of education & $178(33)$ \\
\hline$>12$ years of education & $339(63)$ \\
\hline Father's age [year] & $30.9 \pm 5.5$ \\
\hline \multicolumn{2}{|l|}{ Paternal education: } \\
\hline$<9$ years of education & $24(4)$ \\
\hline 10-12 years of education & $292(54)$ \\
\hline$>12$ years of education & $213(40)$ \\
\hline Marital status (unmarried) & $134(25)$ \\
\hline ETS at the first trimester & 267 (49) \\
\hline ETS at the second trimester & $393(73)$ \\
\hline ETS at the third trimester & $388(72)$ \\
\hline Parental self-reported allergy & $208(38)$ \\
\hline Frequent cleaning (more than 2/week) & $30(8.1)$ \\
\hline
\end{tabular}

ETS - environmental tobacco smoke.

cohort was associated with an increased risk of episodes of wheezing during early childhood [31]. In our study, having a guinea pig in the first trimester of pregnancy increased the risk of wheezing in the first year of life.

Wegienka et al. proved that living with a dog was associated with decreased odds of having $\geq 1$ positive SPT and having eczema [46]. Also according to Thorsteinsdottir et al., the risk of atopic dermatitis was significantly lower in children with domestic dog exposure. The risk of atopic dermatitis decreased in a dose-dependent manner with an increasing number of dogs. The protective effect was restricted to children born to mothers with atopic disorders as no effect was observed in children born to mothers without atopy. Paternal atopic status did not affect the risk of atopic dermatitis [47]. Meta-analysis developed by Pelucchi et al. reported a favourable effect of exposure to dogs and pets on the risk of $A D$ in infants or children, whereas no association emerged with exposure to cats [48]. Our analysis did not show any significant associations between keeping animals at home before and during pregnancy and the occurrence of atopic dermatitis. 
In the second year of life, we did not find a connection between the presence of animals at home before and during pregnancy and the occurrence of food allergies, atopic dermatitis or wheezing. Pregnancy and first months of baby's life are a period of very dynamic development of the baby's immune system and the influence of various factors during this period can condition the development of allergic diseases. Allergy in children is influenced by multiple factors including genetic, socioeconomic, sociodemographic and environmental factors. The relationship between the environment and atopy, as well as between environmental exposure and genetic susceptibility has not been clarified so far. It is known that no single factor determines the development of an allergic disease, it is rather an accumulation of environmental factors.

Domestic animals are one of the most common allergens that children are sensitized to worldwide. The relationship between allergen exposure to animal allergens in pregnancy and the development of allergic symptoms has been investigated in a number of studies but the results are inconsistent. Our study has shown that a dog has a protective effect on the development of food allergy, which may be associated with the beneficial effects of the microbe of this animal. This effect is eliminated in case of having any animal. There is no connection between having animals during pregnancy and the occurrence of allergic symptoms in the second year of life, most probably due to other environmental and genetic factors.

\section{Acknowledgments}

The study was supported by the DEC-2014/15/B/ NZ7/00998 grant from the National Science Centre and by grant 503/2-056-01/503-01 from the Medical University of Lodz, Poland.

\section{Conflict of interest}

The authors declare no conflict of interest.

\section{References}

1. Platts-Mills TA, Vervloet D, Thomas WR, et al. Indoor allergens and asthma: report of the third international workshop. J Allergy Clin Immunol 1997; 100: S2-24.

2. Arshad SH. Does exposure to indoor allergens contribute to the development of asthma and allergy? Curr Allergy Asthma Rep 2010; 10: 49-55.

3. Phillips JF, Lockey RF. Exotic petallergy. J Allergy Clin Immunol 2009; 123: 513-0.

4. Pecquet C. New pets and allergies. Eur J Dermatol 2012; 22: 14-22.

5. Díaz-Perales A, González-de-Olano D, Pérez-Gordo M, Pastor-Vargas C. Allergy to uncommon pets: new allergies but the same allergens. Front Immunol 2013; 4: 492.
6. Eller E, Roll S, Chen CM, et al. Meta-analysis of determinants for pet ownership in 12 European birth cohort on asthma and allergies: a GA2LEN initiative. Allergy 2008; 63: 1491-8.

7. TNS Polska, październik 2014, www.tnsglobal.pl.

8. Zeidler MR, Goldin JG, Kleerup EC, et al. Small airways response to naturalistic cat allergen exposure in subjects with asthma. J Allergy Clin Immunol 2006; 118: 1075-81.

9. Bollinger ME, Eggleston PA, Flanagan E, Wood RA. Cat antigen in homes with and without cats may induce allergic symptoms. J Allergy Clin Immunol 1996; 97: 907-14.

10. Munir AKM, Einarsson R, Schou C, Dreborg SKG. Allergens in school dust. I. The amount of the major cat (Fel d 1 ) and dog (Can $\mathrm{f} 1$ ) allergens in dust from Swedish schools is high enough to probably cause perennial symptoms in most children with asthma who are sensitized to cat and dog. J Allergy Clin Immunol 1993; 91: 1067-74.

11. Liccardi G, D'Amato G, Russo M, et al. Focus on cat allergen (Fel d 1): immunological and aerodynamic characteristics, modality of airway sensitization and avoidance strategies. Int Arch Allergy Immunol 2003; 132: 1-12.

12. D’Amato G, Liccardi G, Russo M, et al. Clothing is a carrier of cat allergens. J Allergy Clin Immunol 1997; 99: 577-8.

13. Liccardi G, Barber D, Russo M, et al. Human hair: an unexpected source of cat allergen exposure. Int Arch Allergy Immunol 2005; 137: 141-4.

14. Liccardi G, Triggiani M, Piccolo A, et al. Sensitization to common and uncommon pets or other furry animals: which may be common mechanisms? Transl Med UniSa 2016; 14: 9-14.

15. Heinrich J, Bedana GB, Zock JP, et al.; the Indoor Working Group of The European Community Health Survey II. Cat allergen level: its determinants and relationship to specific IgE to cat across European centers. J Allergy Clin Immunol 2006; 118: 674-81.

16. Sheikh SI, Pitts J, Ryan-Wenger NA, et al. Environmental exposures and family history of asthma. J Asthma 2016; 53 : 465-70.

17. Sánchez J, Díez S, Cardona R. Pet avoidance in allergy cases: is it possible to implement it? Biomedica 2015; 35: 357-62.

18. Polańska K, Hanke W, Gromadzińska J, et al. Polish mother and child cohort study - defining the problem, the aim of the study and methodological assumption. Int I Occup Med Environ Health 2009; 22: 383-91

19. Polanska K, Hanke W, Jurewicz J, et al. Polish mother and child cohort study (REPRO_PL) - methodology of follow-up of the children. Int J Occup Med Environ Health 2011; 24: 391-8.

20. Polanska K, Krol A, Merecz-Kot D, et al. Maternal stress during pregnancy and neurodevelopmental outcomes of children during the first 2 years of life. J Paediatr Child Health 2017; 53: 263-70.

21. Polańska K, Hanke W, Król A, et al. Polish Mother and Child Cohort Study (REPRO_PL) - Methodology of the follow-up of the children at the age of 7. Int J Occup Med Environ Health 2016; 29: 883-93.

22. Stelmach I, Majak P, Jerzynska J, et al. Cord serum 25hydroxyvitamin D correlates with early childhood viralinduced wheezing. Respir Med 2015; 109: 38-43.

23. Stelmach I, Majak P, Jerzynska J, et al. The effect of prenatal exposure to phthalates on food allergy and early eczema in inner-city children. Allergy Asthma Proc 2015; 36: 72-8.

24. Stelmach I, Grzelewski T, Bobrowska-Korzeniowska M, et al. The role of zinc, copper, plasma glutathione peroxidase enzyme, and vitamins in the development of allergic diseases 
in early childhood: the Polish mother and child cohort study. Allergy Asthma Proc 2014; 35: 227-32.

25. Stelmach I, Bobrowska-Korzeniowska M, Smejda K, et al. Risk factors for the development of atopic dermatitis and early wheeze. Allergy Asthma Proc 2014; 35: 382-9.

26. Koplin JJ, Dharmage SC, Ponsonby AL, et al. Environmental and demographic risk factors for egg allergy in a populationbased study of infants. Allergy 2012; 67: 1415-22.

27. Dunn RR, Fierer N, Henley JB, et al. Home life: factors structuring the bacterial diversity found within and between homes. PLoS One 2013; 8: e64133.

28. Ownby DR, Johnson CC, Peterson EL. Exposure to dogs and cats in the first year of life and risk of allergic sensitization at 6 to 7 years of age. JAMA 2002; 288: 963-72.

29. Fujimura KE, Johnson CC, Ownby DR, et al. Man's best friend? The effect of pet ownership on house dust microbial communities. J Allergy Clin Immunol 2010: 126: 410-2.

30. Fujimura KE, Demoor T, Rauch M, et al. House dust exposure mediates gut microbiome Lactobacillus enrichment and airway immune defense against allergens and virus infection. Proc Natl Acad Sci USA 2014; 111: 805-10.

31. Collin SM, Granell R, Westgarth C, et al. Pet ownership is associated with increased risk of non-atopic asthma and reduced risk of atopy in childhood: findings from a UK birth cohort. Clin Exp Allergy 2015; 45: 200-10.

32. Aichbhaumik N, Zoratti EM, Strickler R, et al. Prenatal exposure to household pets influences fetal immunoglobulin E production. Clin Exp Allergy 2008; 38: 1787-94.

33. Wegienka G, Johnson CC, Havstad S, et al. Lifetime dog and cat exposure and dog-and cat-specific sensitization at age 18 years. Clin Exp Allergy 2011; 41: 979-86.

34. Havstad S, Wegienka G, Zoratti EM, et al. Effect of prenatal indoor pet exposure on the trajectory of total IgE levels in early childhood. J Allergy Clin Immunol 2011; 128: 880-5. e884.

35. Gern JE. Promising candidates for allergy prevention. J Allergy Clin Immunol 2015; 136: 23-8.

36. Tun HM, Konya T, Takaro TK, et al. Exposure to household furry pets influences the gut microbiota of infant at 3-4 months following various birth scenarios. Microbiome 2017; 5: 40.

37. Nagao M, Borres MP, Sugimoto M, et al. Sensitization to secretoglobin and lipocalins in a group of young children with risk of developing respiratory allergy. Clin Mol Allergy 2017; 15: 4.

38. Pyrhönen K, Näyhä S, Läärä E. Dog and cat exposure and respective pet allergy in early childhood. Pediatr Allergy Immunol 2015; 26: 247-55.

39. Pohlabeln H, Jacobs S, Böhmann J. Exposure to pets and the risk of allergic symptoms during the first 2 years of life. J Investig Allergol Clin Immunol 2007; 17: 302-8.

40. Lombardi E, Simoni M, La Grutta S, et al. Effects of pet exposure in the first year of life on respiratory and allergic symptoms in 7-yr-old children. The SIDRIA-2 study. Pediatr Allergy Immunol 2010; 21: 268-76.

41. Lodge CJ, Lowe AJ, Gurrin LC, et al. Pets at birth do not increase allergic disease in at-risk children. Clin Exp Allergy 2012; 42: 1377-85.

42. Brunekreef B, Von Mutius E, Wong G, et al.; ISAAC Phase Three Study Group. Exposure to cats and dogs, and symptoms of asthma, rhinoconjunctivitis, and eczema. Epidemiology 2012; 23: 742-50.

43. Nordlund B, Konradsen JR, Kull I, et al. IgE antibodies to animal-derived lipocalin, kallikrein and secretoglobin are mark- ers of bronchial inflammation in severe childhood asthma. Allergy 2012; 67: 661-9.

44. Eldeirawi K, Kunzweiler C, Combs AM, Persky VW. In utero exposure to pets is associated with asthma and wheezing in Mexican American children. J Asthma 2016; 53: 374-81.

45. Freitas AS, Simoneti CS, Ferraz E, et al. Exposure to high endotoxin concentration increases wheezing prevalence among laboratory animal workers: a cross-sectional study. BMC Pulm Med 2016; 16: 69.

46. Wegienka G, Havstad S, Kim H, et al. Subgroup differences in the associations between dog exposure during the first year of life and early life allergic outcomes. Clin Exp Allergy 2017; 47: 97-105.

47. Thorsteinsdottir S, Thyssen JP, Stokholm J, et al. Domestic dog exposure at birth reduces the incidence of atopic dermatitis. Allergy 2016; 71: 1736-44.

48. Pelucchi C, Galeone C, Bach JF, et al. Pet exposure and risk of atopic dermatitis at the pediatric age: a meta-analysis of birth cohort studies. J Allergy Clin Immunol 2013; 132: 616-22. 